\section{WATCHING WOOD DRY}

For trees, the price of the lightharvesting advantage of height is metastability. Active water loss from the leaf stomata enables sap to be drawn up the tubes of the xylem to a far greater height than vacuum pumping alone could achieve. But it means that the water in the tubes is stretched: under negative pressure and formally in a superheated state. The danger is then cavitation: formation of a gas bubble in the xylem would break the continuous liquid column and disrupt the upward pull.

Plants have evolved precautions against cavitation. That's one reason why the xylem are narrow tubes rather than a single broad artery, exploiting capillarity and reducing the chances of bubble nucleation by confinement. What's more, xylem channels are axially connected segments linked by nanoporous membranes so that bubbles can be kept localized. Microscopic cavities (border pits) in the walls where one xylem segment abuts another serve as bubble traps. And the xylem tubes form an interconnected network to provide redundant paths around any cavitated segment.

Yet cavitation does occur, especially when plants (of any height) are stressed by sub-saturation of moisture in the surrounding soil and atmosphere. This can lead to collapse or breakage (a blocking embolism) of the xylem, which disrupts sap circulation and can be fatal. Cavitation - and recovery from it by fluid release from surrounding cells - has been observed directly $^{1}$, and the resulting release of elastic energy in the xylem can be detected as acoustic emissions ${ }^{2}$, giving rise to a kind of 'plant seismology' for monitoring the integrity of the fluid network.

Exactly how cavitation and drying proceed in a complex network of pores is, however, still a somewhat poorly understood aspect of plant physiology. Partly with that in mind, Vincent et al. have examined the onset and progress of drying in a waterfilled array of interconnected synthetic micropores that mimics some of the key characteristics of the xylem ${ }^{3}$. The circular pores, about $100 \mu \mathrm{m}$ across, are formed within a glass substrate and are interconnected to each other and to the exterior via a thin capping layer of nanoporous silicon. This creates an 'ink bottle' geometry of wide pores linked by narrow necks. The researchers watch how the network empties of water in a sub-saturated atmosphere.

The drying does not simply happen from the outside inwards: there is evidently spontaneous cavitation in some pores, although it is not yet clear if this is homogeneous or heterogeneous (nucleated at the liquid/solid interface). One of the most striking findings is that the emptying happens in quasi-periodic bursts in which many pores empty

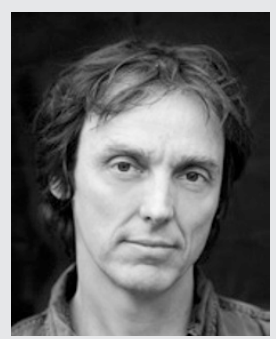

PHILIP BALL

together, separated by quiescent periods of around 15 minutes. There seems to be an analogy with sporadic avalanche phenomena in granular media, where events are due to the build-up and relaxation of stress. There is a similar relaxation here as the release of fluid from the drying pores re-saturates the adjacent pores, creating a temporal periodicity not unlike that seen in reaction-diffusion systems such as the periodic precipitation of Liesegang rings.

The model system may therefore not only elucidate the hydraulic processes and related mechanical failure of both plant tissues and porous rocks but could also represent a new substrate for nonlinear dynamics and pattern formation.

References

1. Brodersen, C. R. et al. Plant Physiol. 154, 1088-1095 (2010).

2. Ponomarekno, A. et al. J. R. Soc. Interface 11, 20140480 (2014).

3. Vincent, O. et al. Phys. Rev. Lett. (in the press); preprint at http://www.arxiv.org/abs/1402.6776 (2014). 